\title{
Determining the critical effective temperature and heat dispersal pattern in monopolar radiofrequency ablation using temperature-time integration
}

\author{
HOW TSENG ${ }^{1}$, SEY-EN LIN ${ }^{2}$, YEN-LIANG CHANG ${ }^{3,4}$, MING-HSU CHEN ${ }^{3}$ and SHIH-HAN HUNG ${ }^{5,6}$ \\ ${ }^{1}$ Department of Biochemistry, Taipei Medical University; ${ }^{2}$ Department of Pathology, \\ Taipei Medical University Hospital, Taipei $110 ;{ }^{3}$ Department of Otolaryngology, Head and Neck Surgery, \\ Cathay General Hospital, Taipei 106; ${ }^{4}$ School of Medicine, Fu-Jen Catholic University, Taipei 242; \\ ${ }^{5}$ Department of Otolaryngology, Taipei Medical University Hospital; ${ }^{6}$ Department of Otolaryngology, \\ School of Medicine, Taipei Medical University, Taipei 110, Taiwan, R.O.C.
}

Received March 31, 2015; Accepted November 25, 2015

DOI: $10.3892 / \mathrm{etm} .2015 .2956$

\begin{abstract}
The radiofrequency ablation (RFA) lesion size is posited to be disproportionate to the total delivered energy, and temperature-time integration (TTI) may have a more critical effect on lesion size. The present study aimed to evaluate this hypothesis by determining the temperature threshold and temperature distribution over tissues during the RFA lesioning process. Using an ex vivo chicken tissue model and an in vivo rabbit model with RFA applied for 2 min under various target temperature settings, the resultant lesions were evaluated histologically using Masson's trichrome stain. The temperature distribution over the tissue during the RFA lesioning process was also determined using a VT02 Visual IR Thermometer. It was revealed that the thermal injury threshold for RFA in the chicken tissues was $\sim 65^{\circ} \mathrm{C}$, but that it ranged from $55-65^{\circ} \mathrm{C}$ in mammals. Using infra-red thermal imaging, the temperature gradient (from the center to the periphery) during the RFA lesioning process demonstrated a uniform heat diffusion pattern. This data supports the proposed hypothesis that TTI is a critical parameter in determining RFA lesion size and can be applied clinically using the following equation: [Target temperature - $\left.55\left({ }^{\circ} \mathrm{C}\right)\right] \mathrm{x}$ time $(\mathrm{sec})$ is proportional to RFA lesion size.
\end{abstract}

\section{Introduction}

Radiofrequency technology has been utilized in medicine for $>100$ years and is frequently used for reducing nasal turbinate,

Correspondence to: Dr Shih-Han Hung, Department of Otolaryngology, Taipei Medical University Hospital, 252 Wu-Hsing Street, Taipei 110, Taiwan, R.O.C.

E-mail: seedturtle@gmail.com

Key words: radiofrequency ablation, temperature threshold, temperature control, temperature time palate and tongue base tissues in the management of nasal obstruction, snoring and obstructive sleep apnea, with promising results (1-7). The radiofrequency energy delivered to the tissue is generally considered to determine lesion size (8). However, according to previous findings, the lesion size of radiofrequency ablation (RFA) is not proportional to the total energy delivered (9). Furthermore, temperature-time integration (TTI) has emerged as a novel predictor of RFA lesion size, improving upon the current metric of total delivered energy when applied in clinical practice. In order to implement TTI as a predictive strategy, it is necessary to know the critical temperature to use as a starting point in the calculation of TTI. The present study proposes that the RFA lesion size may have a more marked correlation with TTI (Fig. 1, shaded area TT') than with total delivered energy. In order to quantify this shaded area, the starting temperature in the $\mathrm{TT}^{\prime}$ area calculations must be determined. This critical temperature is regarded as the temperature threshold for the tissue to be permanently affected or injured by the radiofrequency energy.

In order to deduce accurate TTI parameters, heat dispersal must occur in a smooth pattern, with the highest temperature at the center of the RFA lesion, gradually declining farther from the lesion center. However, previous studies have suggested that tissue heating is not correlated with the RFA probe temperature (10-14) and, in numerous circumstances, the maximal heating temperature appears farther from the RFA probe.

The present study aimed to provide support for the use of TTI as an improved indicator of RFA lesion size, initially through determining the critical temperature threshold for this integration calculation. The temperature distribution over the tissue during the RFA process was also evaluated in order to determine whether the RFA transducer temperature is representative of this thermal injury, and whether heat dispersal follows predictable patterns.

\section{Materials and methods}

Ethics statement. The protocol for the present study was reviewed and approved by the Taipei Medical University 


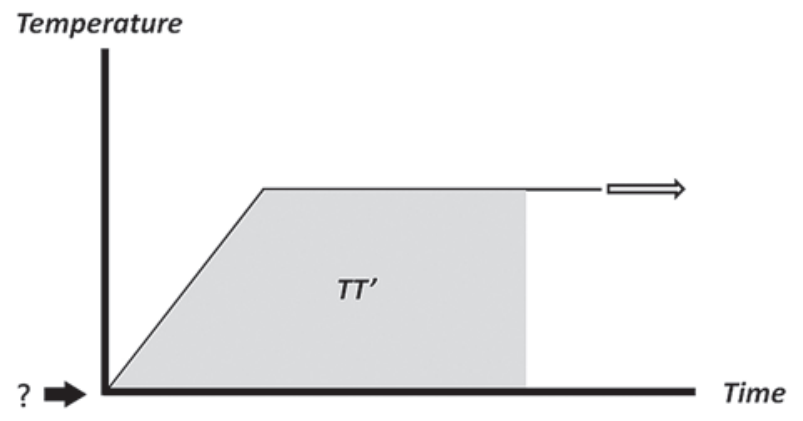

Figure 1. Proposed hypothesis: Lesion size of radiofrequency ablation may demonstrate a more marked correlation with the integration of temperature and time (shaded area, TT') than with total delivered energy. However the starting temperature (threshold) of this calculation is unknown.

Institutional Animal Care and Use Committee (approval no. LAC-2014-0220).

Determination of critical temperature threshold as a starting point for integration calculation. The ex vivo lesioning model used in the present study to create RFA lesions was modified from previous studies (9,15-17). For the in vivo study, three male New Zealand white rabbits (Wei-Hsin Co., New Taipei City, Taiwan), aged 10-15 weeks and weighing 2,500-3,000 g, were used.

Ex vivo lesioning model. Ex vivo fresh chicken breast samples (Sung Ching Commercial Co., Ltd., Taipei, Taiwan) were used as substrates to create the RFA lesions. Previous surface contact methods (15) were modified and the needle was inserted into the chicken muscle tissue with all portions of the active tip embedded. The advantages of using chicken samples are well-described in the previous literature (18). Aside from using chicken tissue, fresh porcine muscle (Sung Ching Commercial Co., Ltd., Taipei, Taiwan) was also tested. The target temperatures were set at $50,55,60$ and $65^{\circ} \mathrm{C}$. For each temperature, RFA was performed in triplicate. Following RFA, the tissues were fixed in formalin for subsequent histological analysis.

In vivo lesioning model. Three male New Zealand white rabbits were used as the in vivo model. Following anesthetization with $50 \mathrm{mg} / \mathrm{kg}$ ketamine and $20 \mathrm{mg} / \mathrm{kg}$ xylazine via intramuscular injection, a wound on the dorsal side of the animal was created by exposing the back muscles. Similar to the aforementioned ex vivo lesioning method, the needle was inserted into the rabbit tissue with all portions of the active tip embedded. The target temperatures were also set at 50 , 55,60 and $65^{\circ} \mathrm{C}$. RFA was performed in triplicate at each temperature. Following RFA, the rabbits were euthanized via intramuscular injection of a double dose of anesthesia, and tissues were excised and fixed in formalin for subsequent histological analysis.

Radiofrequency equipment and RFA. RFA was performed using the temperature-controlled S-1500 RF generator (MedSphere International Holdings, Inc., Shanghai, China). For the RFA procedure, 100-mm, 22-gauge, 10-mm curved, active-tip cannulas (model 10-141221; MedSphere
International Holdings, Inc., Shanghai, China) were used. For the ex vivo and in vivo models, the lesions were created using a power of $25 \mathrm{~W}$. The RFA application time was $2 \mathrm{~min}$, based on the saturation point in a previous study (9).

Histological analysis. Samples were fixed for $24 \mathrm{~h}$ in a $10 \%$ neutral-buffered formalin solution in phosphate-buffered saline ( $\mathrm{pH}$ 7.4) at room temperature. The samples were then washed in distilled water, dehydrated in graded alcohol, embedded in paraffin (Merck, Darmstadt, Germany) and cut into 5-mm sections. Adjacent sections were stained with Masson's trichrome stain (Sigma-Aldrich, St. Louis, MO, USA) to evaluate thermal injury.

Infra-red thermal imaging analysis to measure temperature distribution. Using the ex vivo model, the infra-red thermal image and temperature at the lesion center were captured and recorded at $10-\mathrm{sec}$ intervals using a VT02 Visual IR Thermometer (Fluke, Norwich, UK). For this experiment, the target temperatures were set at 75 and $85^{\circ} \mathrm{C}$, with the RFA application time set to $2 \mathrm{~min}$.

Statistical analysis. In the ex vivo chicken tissue RFA lesioning model, the lesion center temperatures were recorded between 10 and $120 \mathrm{sec}$ and SPSS 17.0 software for Windows was used to compare the data using one-way analysis of variance (SPSS Inc., Chicago, IL, USA). P $<0.05$ was considered to indicate a statistically significant difference.

\section{Results}

The results for the ex vivo model RFA temperature threshold measurement are indicated in Fig. 2A. In the chicken tissues, thermal injury (indicated by purple Trichrome stain; Fig. 2) was not significant until the temperature setting reached $65^{\circ} \mathrm{C}$. In porcine tissues, thermal injury became apparent at temperatures $\geq 55^{\circ} \mathrm{C}$.

The results for the in vivo model RFA temperature threshold measurement are demonstrated in Fig. 2B. When lesions were created in the dorsal muscles of living New Zealand white rabbits, thermal injury was not significant until the target temperature reached $55^{\circ} \mathrm{C}$.

In the ex vivo chicken tissue RFA lesioning model, two target temperatures, 75 and $85^{\circ} \mathrm{C}$, were used. The lesion center temperatures were recorded and the IR thermal images were captured from the beginning of the RFA procedure for $120 \mathrm{sec}$, at 10 -sec intervals. The lesion center rapidly reached $32.8 \pm 0.6$ and $37.8 \pm 1.4^{\circ} \mathrm{C}$, within $10 \mathrm{sec}$ after starting RFA, at 75 and $85^{\circ} \mathrm{C}$, respectively (Fig. 3). Lesion center temperatures reached a plateau subsequent to the rapid temperature increase in the first $10 \mathrm{sec}$. No significant differences were identified between the temperatures recorded at the center of the lesion between 10 and $120 \mathrm{sec}$ at either temperature setting ( 75 and $85^{\circ} \mathrm{C}$, respectively).

The IR thermal color gradient images of the ex vivo chicken tissue RFA lesioning model at the two temperature settings are indicated in Fig. 4A and B. As expected, no thermal differences were detected at $0 \mathrm{sec}$, therefore the whole image was automatically set to the same green color tone. The color gradient images demonstrate the uniform 
CHICKEN

A

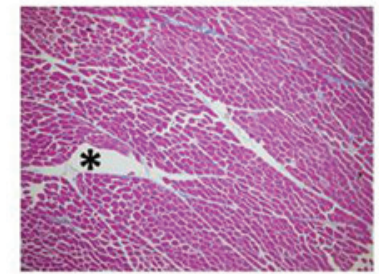

PORCINE

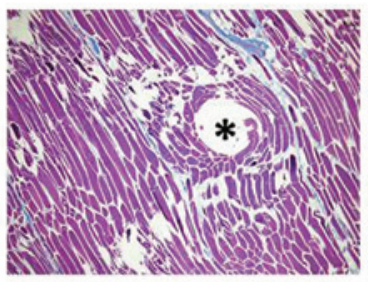

$50^{\circ} \mathrm{C}$
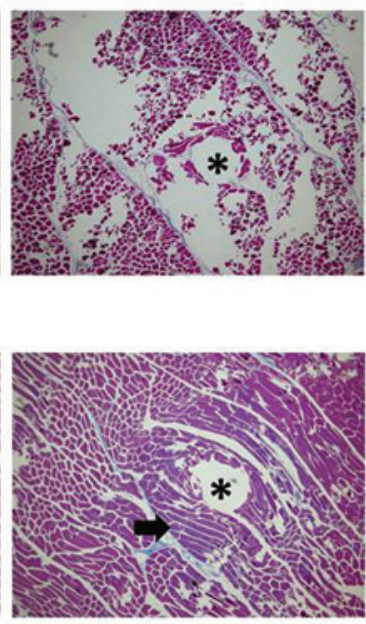

$55^{\circ} \mathrm{C}$

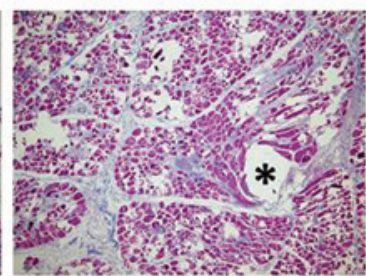

(Trichrome Stain, $\times 40$ )
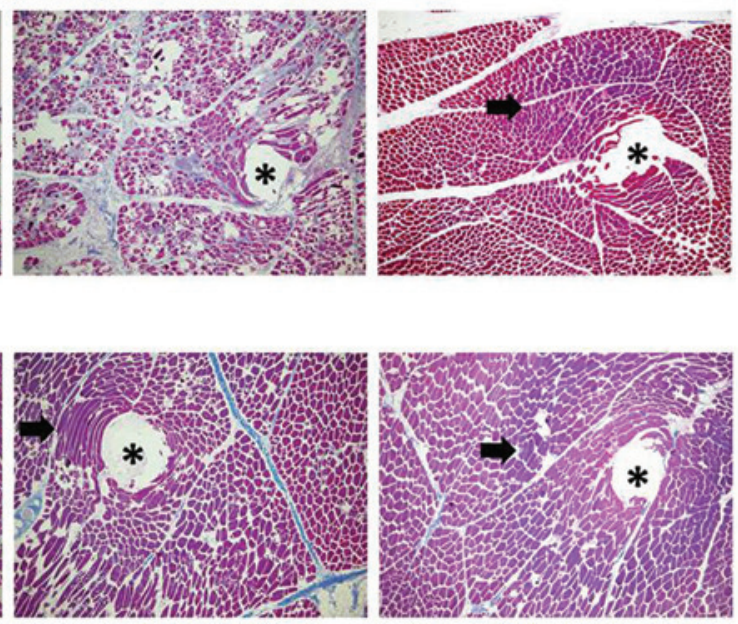

$60^{\circ} \mathrm{C}$

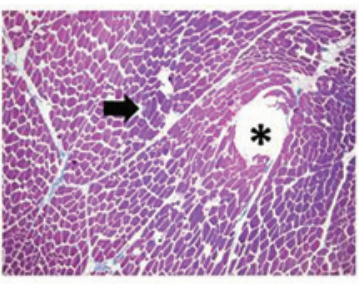

$65^{\circ} \mathrm{C}$

B

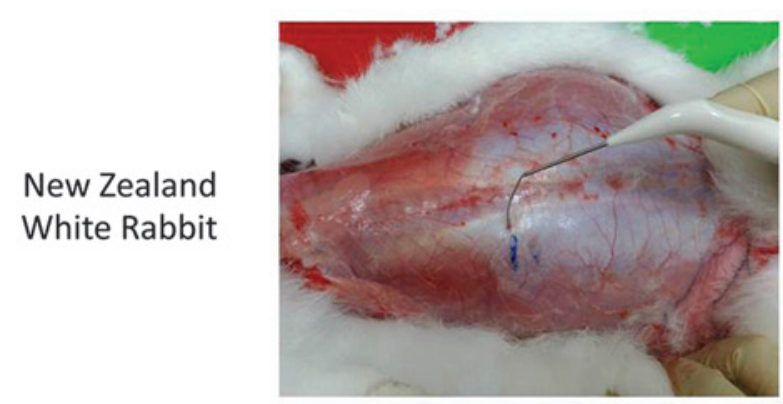

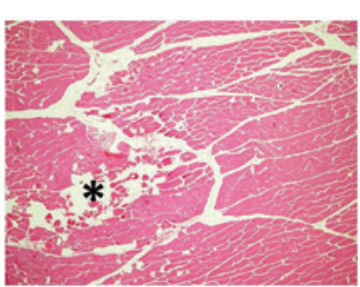

$50^{\circ} \mathrm{C}$

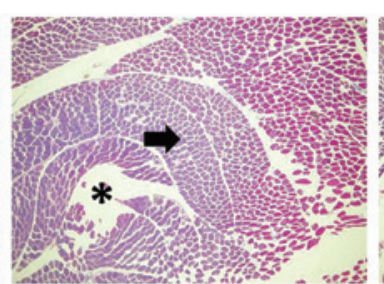

$55^{\circ} \mathrm{C}$

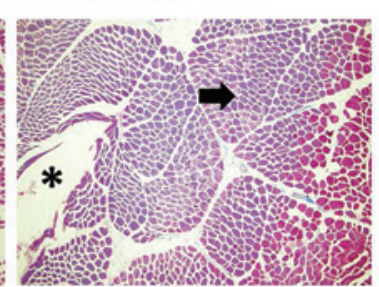

$60^{\circ} \mathrm{C}$
(Trichrome Stain, $\times 40$ )

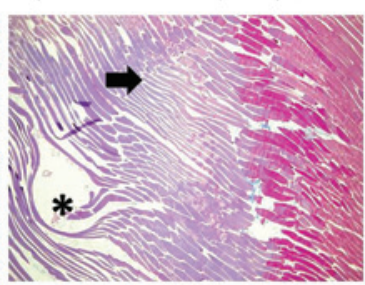

$65^{\circ} \mathrm{C}$

* RFA probe penetration site

$\Rightarrow$ Thermal injury area

Figure 2. (A) Ex vivo lesioning model. In chicken tissues, thermal injury (purple stain under Trichrome staining) was not significant until the target temperature reached $65^{\circ} \mathrm{C}$. In porcine tissues, thermal injury became apparent if the target temperature was $\geq 55^{\circ} \mathrm{C}$. (B) In vivo lesioning model. Upon lesion generation in the dorsal muscles of living New Zealand White rabbits, thermal injury was not significant until the target temperature reached $55^{\circ} \mathrm{C}$. RFA, radiofrequency ablation.

heat diffusion pattern. The temperature gradient reached its equilibrium after $10 \mathrm{sec}$, with no clear gradient pattern change from 10-120 sec. There were no unexpected temperature rises or hot spots throughout the RFA lesioning field.

\section{Discussion}

The present study revealed that the temperature threshold during RFA ranges above the established protein denaturing temperature. The data presented in the current study suggests that the thermal injury threshold for RFA in chicken tissues is $\sim 65^{\circ} \mathrm{C}$. For mammals, the temperature threshold is likely to range from $55-65^{\circ} \mathrm{C}$, presumably closer to $55^{\circ} \mathrm{C}$ for humans due to greater similarity in physiological and histological muscular tissue structures to pigs and rabbits. Furthermore, the present study revealed that, during RFA lesioning, the temperature gradient (from central to peripheral) has a uniform heat diffusion pattern. These data support TTI as a critical parameter in determining RFA lesion size, and suggest that it is clinically applicable to replace joules as a unit of measurement, using the equation: [Target temperature - $\left.55\left({ }^{\circ} \mathrm{C}\right)\right] \mathrm{x}$ time $(\mathrm{sec})$ is proportional to RFA lesion size.

Clinically, if RFA is used to destroy a certain tissue volume (for instance, in otorhinolaryngology to reduce normal tissue), lack of control of thermal energy leads to undesirable therapeutic outcomes. If RFA is used to treat cancer (for example, hepatocellular carcinoma), insufficient RFA may induce additional malignant transformation (19). Control of the RFA procedure is therefore vital to precisely determine how much tissue is destroyed. 


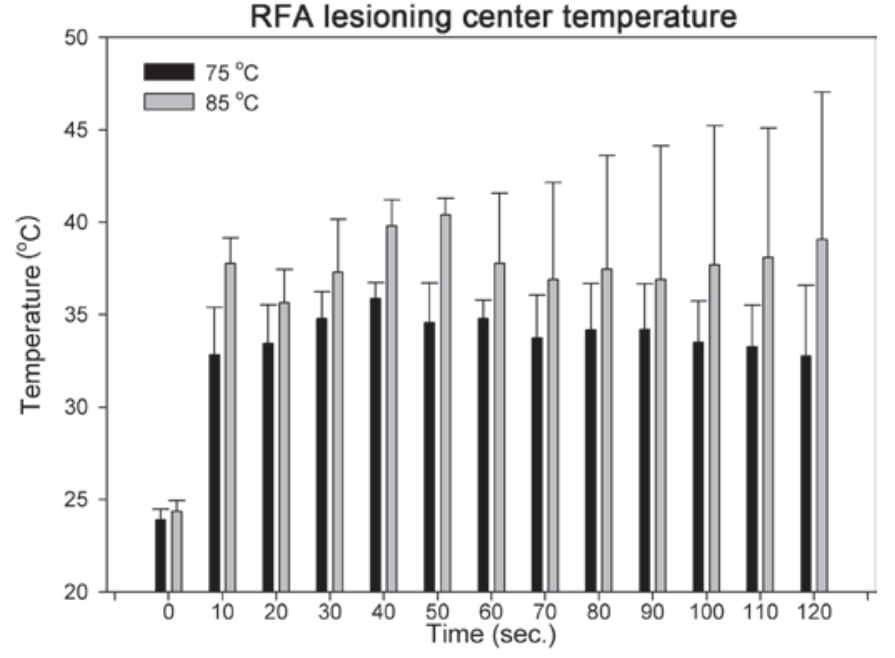

Figure 3. Infra-red thermal imaging analysis under the ex vivo chicken tissue RFA lesioning model. After $10 \mathrm{sec}$ of RFA, the lesion center rapidly reached $32.8 \pm 0.6$ and $37.8 \pm 1.4^{\circ} \mathrm{C}$ under the target temperature settings of 75 and $85^{\circ} \mathrm{C}$, respectively. This temperature rise reached a plateau after $10 \mathrm{sec}$, with no significant differences between the recorded temperatures of the lesion center from 10-120 sec under the two target temperature settings. RFA, radiofrequency ablation.
A

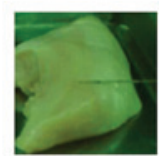

$0 \mathrm{sec}$

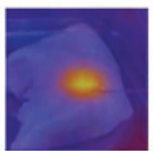

$50 \mathrm{sec}$

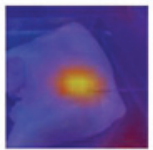

$100 \mathrm{sec}$

B

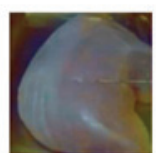

$0 \mathrm{sec}$

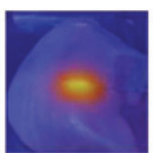

$50 \mathrm{sec}$

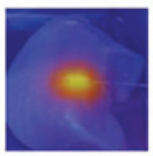

$100 \mathrm{sec}$

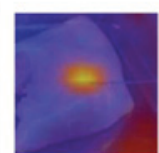

$10 \mathrm{sec}$

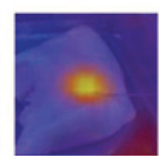

$60 \mathrm{sec}$

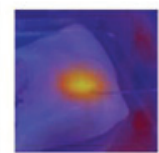

$110 \mathrm{sec}$

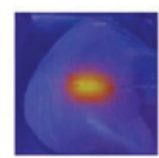

$10 \mathrm{sec}$

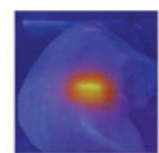

$60 \mathrm{sec}$

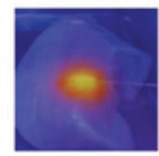

$110 \mathrm{sec}$

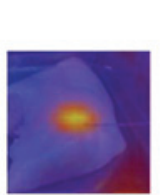

$20 \mathrm{sec}$

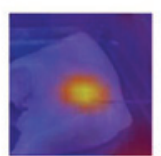

$70 \mathrm{sec}$

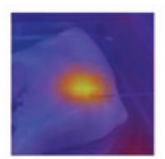

$120 \mathrm{sec}$

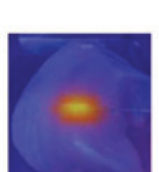

$20 \mathrm{sec}$

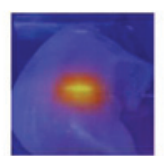

$70 \mathrm{sec}$

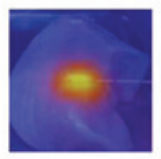

$120 \mathrm{sec}$ $\left(75^{\circ} \mathrm{C}\right.$, ex vivo lesioning)

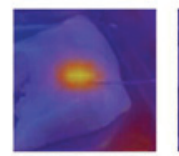

$30 \mathrm{sec}$

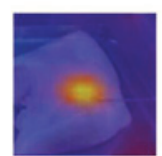

$80 \mathrm{sec}$

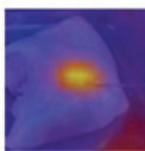

$40 \mathrm{sec}$

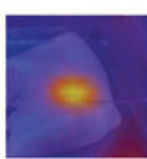

$90 \mathrm{sec}$
( $85^{\circ} \mathrm{C}$, ex vivo lesioning)

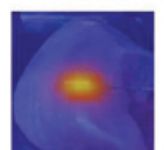

$30 \mathrm{sec}$

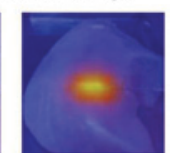

$40 \mathrm{sec}$

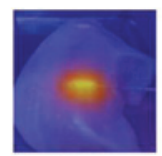

$80 \mathrm{sec}$

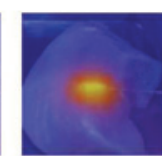

$90 \mathrm{sec}$

Figure 4. Infra-red thermal color gradient images of the chicken tissue ex vivo radiofrequency ablation lesioning model. Under the target temperatures of (A) $75^{\circ} \mathrm{C}$ and (B) $85^{\circ} \mathrm{C}$, uniform heat diffusion patterns were noted. The temperature gradient reached its equilibrium at $10 \mathrm{sec}$, without marked gradient pattern changes from 10-120 sec.

The elevation of cell and tissue temperature is the principal property of radiofrequency currents used to achieve the desired clinical effects (20). There are three basic mechanisms through which radiofrequency currents increase tissue temperature. The first of these is the conversion of electromagnetic energy to mechanical energy. High-frequency alternating currents cause rapid oscillations of electrically-charged particles (ions) within the cellular cytoplasm. Rapid ion movement leads to frictional forces that induce thermal energy production and cause an elevation of intracellular and tissue temperature. The second of these is resistive heating, based on the physical concept of an increased temperature in a resistor due to the current flow. The third mechanism is indirect conductive heat transfer; the increase in tissue temperature leads to the conduction of thermal energy to the adjacent tissue, resulting in the elevation of tissue temperature (21). Thermal injury is known to directly lead to tissue injury and cell death (22).

At a certain temperature, protein denaturation occurs, which may cause cell death. Thermal injury is well-documented in numerous previous cellular studies; for example, Nikfarjam et al (23) documented the importance of focal hyperthermia in the destruction of liver tumors. In a study regarding RFA, Goldberg et al (22) reported that the diameter of tissue coagulation in a pig liver model may be predicted by the local temperature along the exposed electrode, also demonstrating that the diameter of local coagulation necrosis is a function of the mean local temperature.

No coagulation is observed when the local temperature is $<50^{\circ} \mathrm{C}$ in in vitro experiments. Temperatures above this threshold have previously been demonstrated to lead to progressively greater lesion diameter, with a minimum of $1 \mathrm{~cm}$ of necrosis occurring at $71^{\circ} \mathrm{C}$. Additional increases in lesion diameter $(1.4-1.6 \mathrm{~cm})$ have previously been observed at $\sim 90^{\circ} \mathrm{C}$; however, the time setting of this experiment was uniformly set at $6 \mathrm{~min}$, indicating that temperature is important in the prediction of RFA lesion size (22).

Previous evidence in cellular models also suggested that temperature and time are critical parameters to be considered. Moriyama-Gonda et al (24) revealed that a qualitative change in cell death was associated with the degree and duration of thermotherapy of PC-3 cells, from apoptosis to necrosis. Leber et al (25) demonstrated, in a hepatocellular carcinoma cell culture model, that heating resulted in early apoptosis in $20-30 \%$ of HepG2 cells and in $10-15 \%$ of LX-1 cells. Late apoptosis is observed in a large percentage of cells $24 \mathrm{~h}$ after heating at $65^{\circ} \mathrm{C}$ for $15 \mathrm{~min}$ or at $75^{\circ} \mathrm{C}$ for $5 \mathrm{~min}$; by contrast, heating to $65^{\circ} \mathrm{C}$ for $10 \mathrm{~min}$ resulted in only a moderate increase of late apoptotic cells, whilst heating to $55^{\circ} \mathrm{C}$ for $15 \mathrm{~min}$ resulted in a smaller proportion than this of late apoptotic cells. In the present study, the concept of heating time is documented as under a higher temperature, therefore, a shorter duration of exposure is required to achieve comparable thermal injury.

Evidence examined in previous studies supports the current hypothesis of the present study that the TTI may be a better indicator of RFA lesion size. However, there are numerous studies revealing evidence to the contrary. In a previous cardiology study, Petersen et al (10) revealed that, for an established target temperature, power consumption is positively correlated with lesion volume, whereas measured tip temperature is not, suggesting that power is a critical factor 
in determining the lesion size; however, in this previous study, increased convective cooling was achieved through induction of a flow surrounding the electrode tip, increasing lesion dimensions and power consumption.

Nakagawa et al (11), using a canine thigh model, also reported that saline irrigation maintains a low electrode-tissue interface temperature during RFA use at high power, which prevents a rise in electrical impedance, and produces deeper and larger lesions. A similar study by Skrumeda and Mehra (13) demonstrated that irrigated ablation creates larger lesions than standard. Importantly, these contradictory studies have been performed in lesion-irrigating models, in contrast with the present study. In the cardiology study (10), for example, a model was likely used in which RFA would be used to treat arrhythmias.

For the hypothesis in the present study to be applied to solid compartment tissue ablation contexts, including in the management of nasal turbinate reductions, tongue base reductions or liver tumor ablations, it is therefore necessary to ensure that the tip temperature represents a valid reflection of the RFA lesioning area temperature, and that the temperature is dispersed evenly in a smooth gradient pattern. The present study reveals evidence supporting the posited hypothesis and demonstrates that the tip temperature increases rapidly, reaching a plateau within only $10 \mathrm{sec}$; this latter conclusion is important for the application of the suggested equation.

Recalling the RFA lesioning equation proposed in a previous study (9) (target temperature $75^{\circ} \mathrm{C}, \mathrm{y}=0.92 \mathrm{x}-2.2987$; target temperature $65^{\circ} \mathrm{C}, \mathrm{y}=0.3582 \mathrm{x}+2.4539$, where $\mathrm{y}=\mathrm{mm}^{2}$ and $\mathrm{x}=\mathrm{sec}$ ), if the temperature threshold is set at $55^{\circ} \mathrm{C}$ for an established time, the area under $75^{\circ} \mathrm{C}$ is twice as large as the area under $65^{\circ} \mathrm{C}$. At 30 and $40 \mathrm{sec}$, the areas calculated using this equation for 75 and $65^{\circ} \mathrm{C}$ will be $34.5: 16.8$ and 25.2:13.2, respectively, which markedly correlates with the hypothesis equation.

The major limitation of the present study is that the temperature threshold is based on animal tissues; although an in vivo mammal model is used, the equation may not be appropriate for use in human beings. Additional human trials are required to reveal the usefulness of the proposed equation. It should be noted that the equation developed is based on animal muscle tissue and, although it might be useful in the field of otolaryngology for the treatment of sleep apnea, the equation may not be suitable for RFA that is intended for tumor ablations, including the treatment of liver cancer. However, the present study does provide support to this previously proposed hypothesis. The current data suggest that the use of temperature-controlled radiofrequency devices with a target temperature set to $>65^{\circ} \mathrm{C}$ should be lowered to the temperature threshold of $55^{\circ} \mathrm{C}$ for RFA in otorhinolaryngology. The current study also indicates that the RFA time should be considered a reliable parameter in determining the desired tissue ablation volume.

The thermal injury threshold for RFA in chicken tissues is $\sim 65^{\circ} \mathrm{C}$ and ranges from $55-65^{\circ} \mathrm{C}$ for mammals. During RFA lesioning, the temperature gradient from the center to the periphery demonstrates a uniform heat diffusion pattern. TTI appears to be a critical parameter in determining RFA lesion size and is clinically applicable as the following equation [Target temperature $-55\left({ }^{\circ} \mathrm{C}\right)$ ] $\mathrm{x}$ time $(\mathrm{sec})$ is proportional to RFA lesion size.

\section{Acknowledgements}

Thepresentstudywassupported by the TaipeiMedicalUniversity Hospital Research Fund (grant no. 103TMU-TMUH-20).

\section{References}

1. Pirsig W: Has tongue base reduction with radiofrequency energy in sleep apnea syndrome been adequately evaluated? HNO 49: 501-502, 2001 (In German).

2. Stuck BA, Maurer JT and Hörmann K: Tongue base reduction with radiofrequency energy in sleep apnea. HNO 49: 530-537, 2001 (In German).

3. Blumen MB, Dahan S, Fleury B, Hausser-Hauw C and Chabolle F: Radiofrequency ablation for the treatment of mild to moderate obstructive sleep apnea. Laryngoscope 112: 2086-2092, 2002.

4. Li KK, Powell N and Riley R: Radiofrequency thermal ablation therapy for obstructive sleep apnea. Oral Maxillofac Surg Clin North Am 14: 359-363, 2002.

5. Powell NB, Riley RW, Troell RJ, Li K, Blumen MB and Guilleminault C: Radiofrequency volumetric tissue reduction of the palate in subjects with sleep-disordered breathing. Chest 113: 1163-1174, 1998.

6. Powell NB, Riley RW and Guilleminault C: Radiofrequency tongue base reduction in sleep-disordered breathing: A pilot study. Otolaryngol Head Neck Surg 120: 656-664, 1999.

7. Lin HC, Lin PW, Friedman M, Chang HW, Su YY, Chen YJ and Pulver TM: Long-term results of radiofrequency turbinoplasty for allergic rhinitis refractory to medical therapy. Arch Otolaryngol Head Neck Surg 136: 892-895, 2010.

8. Eick OJ: Temperature controlled radiofrequency ablation. Indian Pacing Electrophysiol J 2: 66-73, 2002.

9. Chang YL, Tseng TM, Chen PY, Lin CJ and Hung SH: Using temperature-time integration as a critical parameter in using monopolar radiofrequency ablations. Eur Arch Otorhinolaryngol 271: 1973-1979, 2014.

10. Petersen HH, Chen X, Pietersen A, Svendsen JH and Haunsø S: Lesion size in relation to ablation site during radiofrequency ablation. Pacing Clin Electrophysiol 21: 322-326, 1998.

11. Nakagawa H, Yamanashi WS, Pitha JV, Arruda M, Wang X, Ohtomo K, Beckman KJ, McClelland JH, Lazzara R and Jackman WM: Comparison of in vivo tissue temperature profile and lesion geometry for radiofrequency ablation with a saline-irrigated electrode versus temperature control in a canine thigh muscle preparation. Circulation 91: 2264-2273, 1995.

12. Dorwarth U, Fiek M, Remp T, Reithmann C, Dugas M, Steinbeck G and Hoffmann E: Radiofrequency catheter ablation: Different cooled and noncooled electrode systems induce specific lesion geometries and adverse effects profiles. Pacing Clin Electrophysiol 26: 1438-1445, 2003.

13. Skrumeda LL and Mehra R: Comparison of standard and irrigated radiofrequency ablation in the canine ventricle. J Cardiovasc Electrophysiol 9: 1196-1205, 1998.

14. Petersen HH, Chen X, Pietersen A, Svendsen JH and Hauns $\varnothing$ S: Tissue temperatures and lesion size during irrigated tip catheter radiofrequency ablation: An in vitro comparison of temperature-controlled irrigated tip ablation, power-controlled irrigated tip ablation, and standard temperature-controlled ablation. Pacing Clin Electrophysiol 23: 8-17, 2000.

15. Provenzano DA, Lassila HC and Somers D: The effect of fluid injection on lesion size during radiofrequency treatment. Reg Anesth Pain Med 35: 338-342, 2010.

16. Provenzano DA, Liebert MA and Somers DL: Increasing the $\mathrm{NaCl}$ concentration of the preinjected solution enhances monopolar radiofrequency lesion size. Reg Anesth Pain Med 38: 112-123, 2013.

17. Provenzano DA, Watson TW and Somers DL: The interaction between the composition of preinjected fluids and duration of radiofrequency on lesion size. Reg Anesth Pain Med 40: 112-124, 2015.

18. Ahmed M, Liu Z, Afzal KS, Weeks D, Lobo SM, Kruskal JB, Lenkinski RE and Goldberg SN: Radiofrequency ablation: Effect of surrounding tissue composition on coagulation necrosis in a canine tumor model. Radiology 230: 761-767, 2004.

19. Obara K, Matsumoto N, Okamoto M, Kobayashi M, Ikeda H, Takahashi H, Katakura Y, Matsunaga K, Ishii T, Okuse C, et al: Insufficient radiofrequency ablation therapy may induce further malignant transformation of hepatocellular carcinoma. Hepatol Int 2: 116-123, 2008. 
20. Ihnat $P$, Ihnat Rudinska L and Zonca P: Radiofrequency energy in surgery: state of the art. Surg Today 44: 985-991, 2014.

21. Massarweh NN, Cosgriff N and Slakey DP: Electrosurgery: History, principles, and current and future uses. J Am Coll Surg 202: 520-530, 2006.

22. Goldberg SN, Gazelle GS, Halpern EF, Rittman WJ, Mueller PR and Rosenthal DI: Radiofrequency tissue ablation: Importance of local temperature along the electrode tip exposure in determining lesion shape and size. Acad Radiol 3: 212-218, 1996.
23. Nikfarjam M, Muralidharan V and Christophi C: Mechanisms of focal heat destruction of liver tumors. J Surg Res 127: 208-223, 2005.

24. Moriyama-Gonda N, Igawa M, Shiina H, Urakami S, Shigeno K and Terashima M: Modulation of heat-induced cell death in PC-3 prostate cancer cells by the antioxidant inhibitor diethyldithiocarbamate. BJU Int 90: 317-325, 2002.

25. Leber B, Mayrhauser U, Leopold B, Koestenbauer S, Tscheliessnigg K, Stadlbauer V and Stiegler P: Impact of temperature on cell death in a cell-culture model of hepatocellular carcinoma. Anticancer Res 32: 915-921, 2012. 